\title{
Collision Avoidance for Unmanned Surface Vehicles based on COLREGS
}

\author{
Jiayuan Zhuang \\ College of Shipbuilding Engineering \\ Harbin Engineering University \\ Harbin,China \\ zhuangjiayuan@vip.163.com
}

\author{
Richard Bucknall \\ Department of Mechanical Engineering \\ University College London \\ London, UK \\ r.bucknall@ucl.ac.uk
}

\author{
Jing Luo \\ College of Shipbuilding Engineering \\ Harbin Engineering University \\ Harbin,China \\ luojing@hrbeu.edu.cn \\ Hanbing Sun \\ College of Shipbuilding Engineering \\ Harbin Engineering University \\ Harbin,China \\ sunhanbing@hrbeu.edu.cn
}

\author{
Yuanchang Liu \\ Department of Mechanical Engineering \\ University College London \\ London, UK \\ yuanchang.liu@ucl.ac.uk
Cheng Huang
Wuchang Shipbuilding Industry Group Co., Ltd
Wuhan,China
huangcheng@cimoe.com

\begin{abstract}
Unmanned surface vehicles (USVs) are becoming increasingly vital in a variety of maritime applications. The development of a real-time autonomous collision avoidance system is the pivotal issue in the study on USVs, in which the reliable collision risk detection and the adoption of a plausible collision avoidance maneuver play a key role. Existing studies on this subject seldom integrate the International Regulations for Preventing Collisions at Sea 1972 (COLREGS) guidelines. However, in order to ensure maritime safety, it is of fundamental importance that such a regulation should be obeyed at all times. In this paper, an approach of real-time collision avoidance has been presented with the compliance with the COLREGS rules been successfully integrated for USV. The approach has been designed in a way that through the judgment of the collision situation, the velocity and heading angle of the USV are changed to complete the collision avoidance of the obstacle. A strategy with reference obstacle is proposed to deal with the multiple moving obstacles situation. A number of simulations have been conducted in order to confirm the validity of the theoretic results obtained. The results show that the algorithms can sufficiently deal with complex traffic environments and that the generated practical path is suitable for USVs.
\end{abstract}

Keywords-Unmanned surface vehicles (USVs), COLREGS, velocity obstacle, collision avoidance

\section{INTRODUCTION}

USVs are powerful instruments used in a wide variety of maritime missions in civil, military or research applications including oceanography, remote sensing, weapons delivery, force multipliers, environmental monitoring, surveying, antisubmarine warfare, surface warfare, electronic warfare and maritime interdiction operations support, as illustrated in studies [1-4]. One of the main challenges for USVs is the avoidance of (static and dynamic) obstacles. A Kalman filterbased predictive path planning algorithm is proposed by Yuanchang Liu et al.[5] The algorithm has been designed to predict the trajectories of moving ships, and the USV's own position in real time and accordingly assesses collision risk.

After confirming a potential conflict, the next step is to adopt a timely avoidance maneuver. Previous studies have attempted to resolve this issue; Svec [6], for instance, employed a nominal trajectory planner to generate a collision free trajectory between the current state of a USV and its motion goal and, in the work of Soltan [7], ordinary differential equations were used to define transitional trajectories able to avoid obstacles. Phanthong [8], meanwhile, adopted a numerical solution procedure based on an An algorithm to compute near-optimal paths; he then used a robust real-time path re-planning technique to avoid moving obstacles. Nevertheless, these studies focused solely on the computation of a collision-free path without respecting International Regulations for Preventing Collisions at Sea 1972 (COLREGS) compliancy

The International Maritime Organization (IMO) established the COLREGS[9] as a universal and definitive guide for executing standard avoidance maneuvers. However, as highlighted in a recent survey [10], the vast majority of research that has been conducted in the area of obstacle avoidance has failed to integrate these rules. So, as Statheros et al. [11] report, $56 \%$ of all collisions at sea involve a violation of the COLREGS rules. In consequence, it is important for USV to maintain a strict adherence to the COLREGS at all times.

There are a few studies that successfully integrate the COLREGS rules into collision avoidance techniques for USVs. One significant study can be found in the work undertaken by Benjamin [12], who applied Interval Programming in a behavior-based control framework to represent the navigation rules for safe navigation. Tam et al. [13] proposed a deterministic path planning algorithm able to compute a practical and COLREGS compliant navigation path so that output consistency can be maintained. An automatic obstacle avoidance system for USV emerged in relevant literature [14], in which the R-RAn method was developed for path re-planning when a ship is confronted with multiple approaching vessels. The proposed R-RAn algorithm is able to incorporate the necessary COLREGS rules. Similarly, Naeem et al. [1] established a collision avoidance strategy that consists of a real-time path-planning scheme using the biased line-of-sight method and an offline trajectory generation with a DPSS algorithm able to produce COLREGS-compliant paths. Breitsprecher [15] posited a decision rule induction algorithm in order to build a COLREGS knowledge database and implement it to form an expert decision support system. A framework for a decision-action execution model was introduced by Perera [16] to facilitate intelligent collision avoidance while respecting the COLREGS rules, in which the Fuzzy-Bayesian-based decision/action formulation process was used to avoid a situation in which a complex collision occurred. Yuxin Zhao et al.[17] also presented a fuzzy logicbased intelligent decision making system to improve the safety of marine vessels. Kuwata et al. [18] put forward a 
meritorious approach to motion planning in which the Velocity Obstacles (VO) algorithm [19] was adopted to generate a collision-free path while obeying the COLREGS rules. This approach has the advantage of guaranteeing the safe navigation of USV in cluttered environments. However, this approach, based as it is on the assumption that the vessels encountered are passive, while, in fact, in an actual marine situation, the encountered vessels also sense their ambience and change their trajectories accordingly. As a consequence, the path generated by this approach may in fact be unreliable. Chee Kuang Tam et al. [20] describes the development of a deterministic path planning algorithm that computes a practical and COLREGS compliant navigation path for vessels which are on a collision course. The main advantage of a deterministic algorithm over a stochastic algorithm is its algorithmic completeness. This is an important property in navigation where the behavior of the algorithm can be assured, therefore improving the confidence of the navigation path generated. Furthermore, it will work with other vessels that are without such path planning systems since its computations are based on COLREGS regulations as well as common good seamanship practice.

In this paper, we intend to develop a collision avoidance algorithm that complies with the COLREGS rules for USV. The purpose of this algorithm is to make a plausible decision concerning collision avoidance that is COLREGS compliant, with the reactive avoidance action of the threatening vessel being simultaneously taken into account. Firstly, determine the encounter situation between USV and obstacles, in general, there are three kinds of encounters situation: Overtaking, Head-on, Crossing. Then carry out collision avoidance operation according to different encounter situation and extern to the multiple obstacles situation.

\section{COLREGS}

COLREGS consists of three parts, of which 'Part A: General' describes the applicability and responsibilities of the regulations. 'Part B:Steering and Sailing Rules' pertain to navigational practice, whereas 'Part C: Lights and Shapes' addresses the topics of various signals and use of lighting. They are paired with the Inland Navigation Rules, which exhibit similar protocols, with only marginal differences applying only to inland waters and lakes. Due to recent important advances in unmanned technology, the Navigation Safety Advisory Council (NAVSAC) has put forward a proposal for amending COLREGS to accommodate UUV and USV operations. Current law does not specifically address the use of unmanned vehicles in a marine environment. The use of such vehicles presents a risk of injury and property damage. A natural and prudent solution is for the designer to follow COLREGS until more precise law regulating USVs is enacted. Based on the COLREGS, the Fig.1 shows the definition of USVs collision scenario.

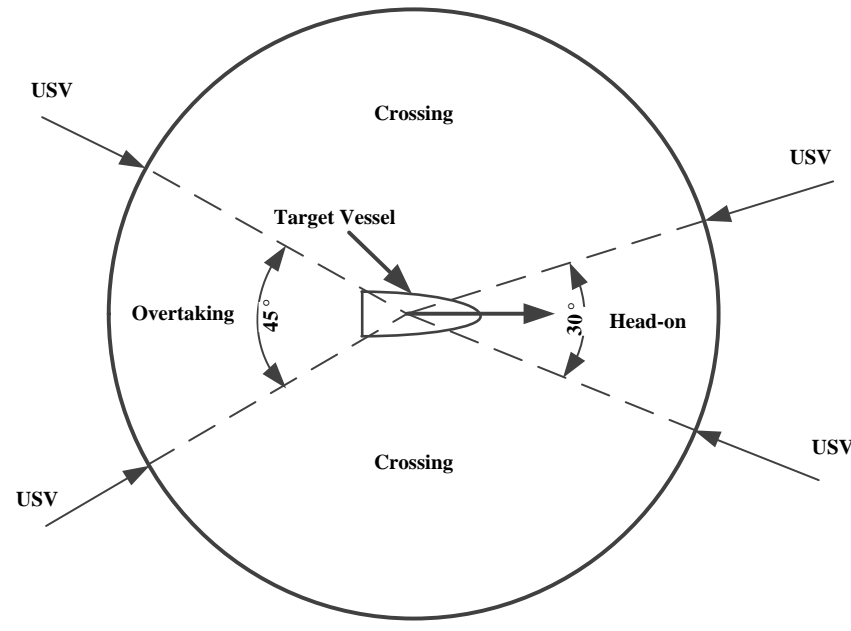

Fig. 1 Collision scenario definition

The three primary rules are as follows: Rule 13: Overtaking, Rule 14: Head-on and Rule 15: Crossing. The rules are illustrated for clarity in Fig. 2 to Fig. 4. All avoidance actions must be obvious and taken well in advance. The overtaking rule (Fig 2) stipulates that a vessel may pass on either the port (left) or starboard (right) side but must issue the appropriate signal. In a Head-on encounter (Fig. 3), when powered vessels approach each other, they must pass port to port by making respective starboard maneuvers. When two vessels are crossing (Fig. 4), the powered vessel that has the other on its starboard side must give way by waiting until it has passed or by crossing abaft of (behind) the stand-on vessel.

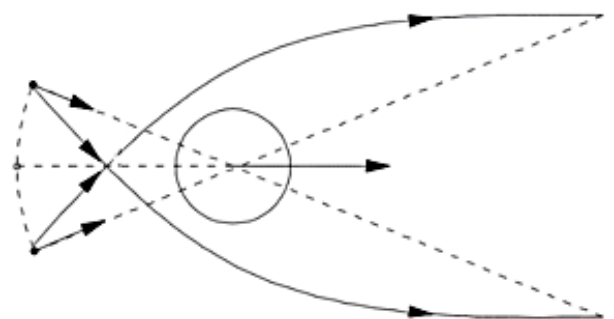

Fig. 2 Rule 13: Overtaking.

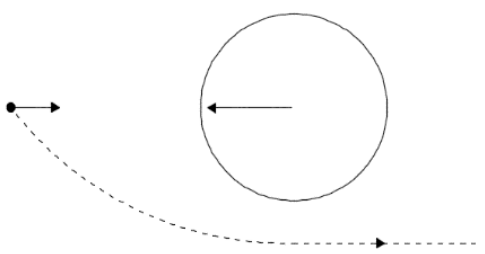

Fig. 3 Rule 14: Head-on.

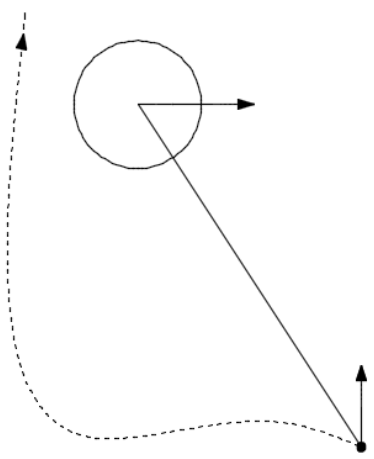

Fig. 4 Rule 15: Crossing 


\section{COLLISION RISK ASSESSMENT}

In autonomous USV navigation, judgments on and appropriate avoidance measures for possible collisions are necessary. Therefore, an important part of the USV intelligent collision avoidance is assessing the collision situation accurately. This paper defined the collision model as follows (Fig. 5 and Fig. 6).

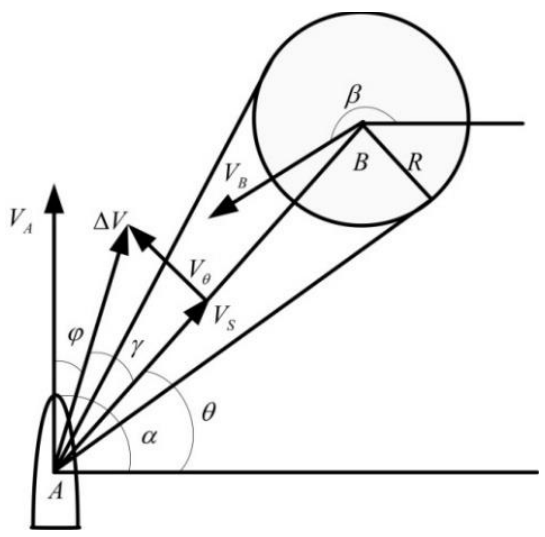

Fig. 5. The collision model of USV and obstacle

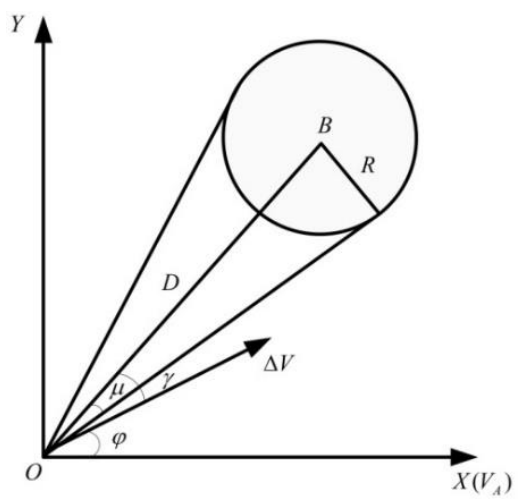

Fig. 6. The relative coordinate

In Fig. 5, A is a point object that represents the USV and $\mathrm{B}$ is a circle of radius $\mathrm{R}$ with its center representing an obstacle. The velocities of $\mathrm{A}$ and $\mathrm{B}$ are denoted by $V_{A}$ and $V_{B}$, respectively. The behavior of LOS is characterized by Eq. (1):

$$
\left\{\begin{array}{l}
V_{s}=V_{A} \cos (\alpha-\theta)-V_{B} \cos (\beta-\theta) \\
V_{\theta}=V_{A} \sin (\alpha-\theta)-V_{B} \sin (\beta-\theta)
\end{array}\right.
$$

where $V_{s}$ and $V_{\theta}$ are the relative components along and perpendicular to the LOS, respectively. $\Delta V$ is the relative velocity of $\mathrm{A}$ with respect to $\mathrm{B}\left(\Delta V=V_{A}-V_{B}\right) . \gamma$ is the angle between $\Delta V$ and LOS, and $\varphi$ is the angle between $\Delta V$ and $V_{A}$.

In Fig. 6, the USV is the coordinate origin denoted by $O$ and the $\mathrm{X}$-axis is defined by the direction of velocity $V_{A}$. The obstacle encountered by the USV is denoted by $B$. The distance between the USV and the obstacle is $D$, which can be measured by radar. $R$ is the safe distance, and $\mu$ denotes the safe or collision angle, which is calculated using Eq. (2):

$$
\mu=\tan ^{-1}\left(\frac{R}{\sqrt{D^{2}-R^{2}}}\right)
$$

If angle $\gamma$ is equal to the outside angle $\mu$, the USV would be safe and collision_flag $=1$; otherwise, the USV will likely collide with the obstacle.

$$
\begin{cases}\gamma \leq \mu & \text { collision_flag }=1 \\ \gamma>\mu & \text { collision_flag }=0\end{cases}
$$

\section{COLLISION AVOIDANCE ALGORITHM}

USVs operate at high speeds and have great maneuverability, so there is a high real-time requirement for a collision avoidance algorithm. When the Automatic Identification System (AIS) or marine radar perceives possible collision obstacles, the USV needs to take reasonable evasive actions for collision avoidance. In view of these requirements, an approach based on the velocity obstacle (Vobstacle) concept can be applied. This approach enables efficient selection of a single velocity and head angle for the USV to would avoid any number of moving obstacles (if such a solution exists)

Fiorini and Shiller [19] proposed the V-obstacle concept to realize real-time motion planning of a robot in dynamic environments. They applied this method to online collision and moving obstacle avoidance, as well as real-time nonlinear velocity obstacle planning. Other studies also investigated and applied the V-obstacle avoidance theory extensively[18][21].

From Fig. 5 and Eq. (1) the Eq. (4) can be obtained

$$
\tan \gamma=\frac{V_{\theta}}{V_{s}}=\frac{V_{A} \sin (\alpha-\theta)-V_{B} \sin (\beta-\theta)}{V_{A} \cos (\alpha-\theta)-V_{B} \cos (\beta-\theta)}
$$

A function of the magnitude and direction angle of the velocity was defined as

$$
\tan \gamma=f\left(V_{A}, \alpha, V_{B}, \beta\right)
$$

Then obtain $\gamma=\tan ^{-1} f\left(v_{a}, \alpha, v_{b}, \beta\right)$

$$
d \gamma=\frac{1}{1+f^{2}} d f
$$

where

$$
\frac{1}{1+f^{2}}=\frac{K^{2}}{V_{A}^{2}+V_{B}^{2}-2 V_{A} V_{B} \cos (\alpha-\beta)}
$$$$
K^{2}=\left[V_{B} \cos (\beta-\theta)-V_{A} \cos (\alpha-\theta)\right]^{2} \text {. }
$$

$$
d f=\frac{\partial f}{\partial V_{A}} d V_{A}+\frac{\partial f}{\partial \alpha} d \alpha+\frac{\partial f}{\partial V_{B}} d V_{B}+\frac{\partial f}{\partial \beta} d \beta
$$

Because the USV only could be controlled and not the obstacle at any given time and because the variation in the obstacle's magnitude and direction of velocity is negligible in a very small time interval, the variation can be approximated to zero, and Eq. (6) can be approximated to

$$
d f=\frac{\partial f}{\partial V_{A}} d V_{A}+\frac{\partial f}{\partial \alpha} d \alpha=d V_{A}
$$

where

$$
\frac{\partial f}{\partial V_{A}}=\frac{-V_{B} \sin (\alpha-\beta)}{K^{2}}
$$

$\frac{\partial f}{\partial \alpha}=\frac{V_{A}\left[V_{A}-V_{B} \cos (\alpha-\beta)\right]}{K^{2}}$. 
Hence, Eq. (6) can be rewritten as

$d \gamma=\frac{-V_{B} \sin (\alpha-\beta) d V_{A}+V_{A}\left[V_{A}-V_{B} \cos (\alpha-\beta)\right] d \alpha}{V_{A}^{2}+V_{B}^{2}-2 V_{A} V_{B} \cos (\alpha-\beta)}$

Instead of the differential of the difference $\gamma$, the equation above can be rewritten as

$\Delta \gamma=\frac{-V_{B} \sin (\alpha-\beta) \Delta V_{A}+V_{A}\left[V_{A}-V_{B} \cos (\alpha-\beta)\right] \Delta \alpha}{V_{A}^{2}+V_{B}^{2}-2 V_{A} V_{B} \cos (\alpha-\beta)}$

The relationship between the velocities is shown in Fig. 7:

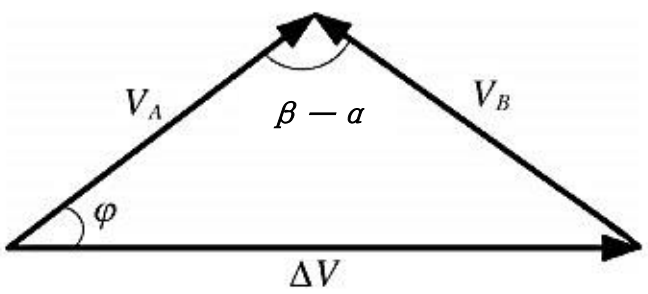

Fig. 7. The relation of velocities

$$
\Delta \gamma=\frac{-V_{B} \sin (\alpha-\beta) \Delta V_{A}+V_{A}\left[V_{A}-V_{B} \cos (\alpha-\beta)\right] \Delta \alpha}{V_{A}^{2}+V_{B}^{2}-2 V_{A} V_{B} \cos (\alpha-\beta)}
$$

From Fig. 7, we get:

$$
\begin{gathered}
V_{B} \sin (\alpha-\beta)=-\Delta V \sin \varphi \\
V_{A}-V_{B} \cos (\alpha-\beta)=\Delta V \cos \varphi \\
V_{A}^{2}+V_{B}^{2}-2 V_{A} V_{B} \cos (\alpha-\beta)=\Delta V^{2}
\end{gathered}
$$

Then $\quad \Delta \gamma=\frac{\Delta V_{A} \sin \varphi+V_{A} \Delta \alpha \cos \varphi}{\Delta V}$

And

$$
V_{A} \Delta \alpha=-\tan \varphi \Delta V_{A}+\frac{\Delta V \Delta \gamma}{\cos \varphi}
$$

$\Delta V_{A}$ is the variation of relative velocity, and $\Delta \alpha$ is the variation of heading angle magnitude. $V_{A} \Delta \alpha$ is approximately perpendicular to the direction of $\Delta V_{A}$.

$$
V_{A} \Delta \alpha=\cot \varphi \Delta V_{A}
$$

From Fig. 6 and Eq. (16), angle $\gamma$ from angle $\mu$ need to subtract; the variation range of $\Delta \gamma$ is called the collision field.

$$
\left\{\begin{array}{l}
\Delta \gamma=\mu-\gamma \\
\Delta \gamma=\mu+\gamma
\end{array}\right.
$$

The variations of the USV's velocity and head angle magnitude for each time interval can be obtained as follows:

$$
\left\{\begin{array}{l}
V_{A} \Delta \alpha=\Delta V \Delta \gamma \cos \varphi \\
\Delta V_{A}=\Delta V \Delta \gamma \sin \varphi
\end{array}\right.
$$

\section{COLLISION AVOIDANCE STRATEGY}

The purpose of this paper is to make a plausible decision concerning collision avoidance that is COLREGS compliant, with the reactive avoidance action of the threatening obstacle being simultaneously taken into account. Firstly, the collision avoidance strategy based on single moving obstacle is given, and then extended to the complex situation of multiple moving obstacles.

\section{A. Singal moving obstacle}

As shown in Fig. 5, counterclockwise direction is the direction of angle increase in polar coordinates.

1) Overtaking: When the heading angle of USV is less than the heading angle of obstacle, the USV should adjust its speed and heading angle then pass through the right side of obstacle moving direction. It is shown in Fig.8 and Eq. (19).

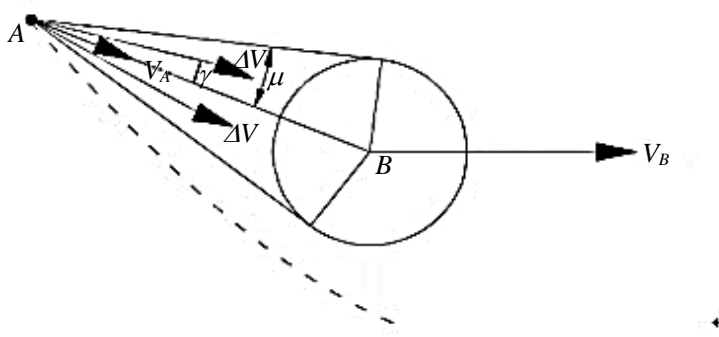

Fig. 8. The strategy of Overtaking ( $\alpha \leq \beta$ )

$$
\begin{cases}\Delta \gamma=-(\mu+\gamma) & \alpha \leq \beta, \phi \geq \theta \\ \Delta \gamma=-(\mu-\gamma) & \alpha \leq \beta, \phi<\theta\end{cases}
$$

Where $\phi$ is the heading angle of $\Delta V$.

When the heading angle of USV is larger than the heading angle of obstacle, the USV should adjust its speed and heading angle then pass through the left side of obstacle moving direction. It is shown in Fig. 9 and Eq. (20).

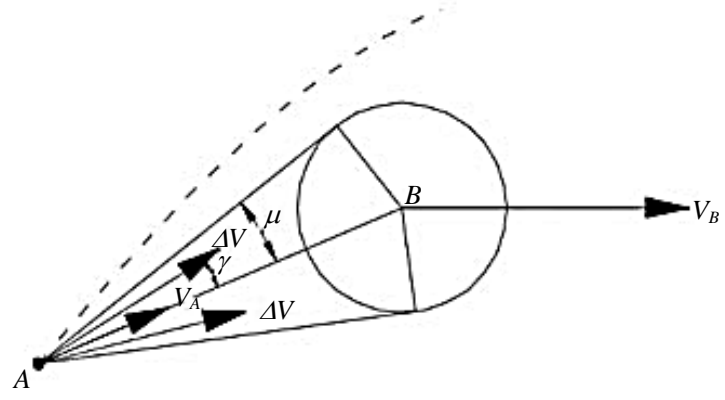

Fig. 9 The strategy of Overtaking $(\alpha>\beta)$

$$
\begin{cases}\Delta \gamma=\mu-\gamma & \alpha>\beta, \phi \geq \theta \\ \Delta \gamma=\mu+\gamma & \alpha>\beta, \phi<\theta\end{cases}
$$

2) Head-on: The USV should adjust its speed and heading and pass through the left side of obstacle moving direction. It is shown in Fig. 10 and Eq. (21).

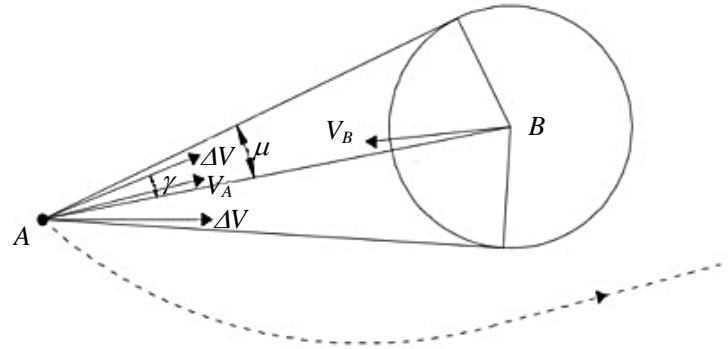

Fig. 10 The strategy of Head-on 


$$
\begin{cases}\Delta \gamma=-(\mu+\gamma) & \phi \geq \theta \\ \Delta \gamma=-(\mu-\gamma) & \phi<\theta\end{cases}
$$

3) Crossing: The USV should adjust its speed and heading and pass through the backward of obstacle moving direction. This situation can be divided into two cases: $\phi$ decreases or increases. These are shown in Fig. 11, Fig, 12and Eq. (22), Eq. (23).

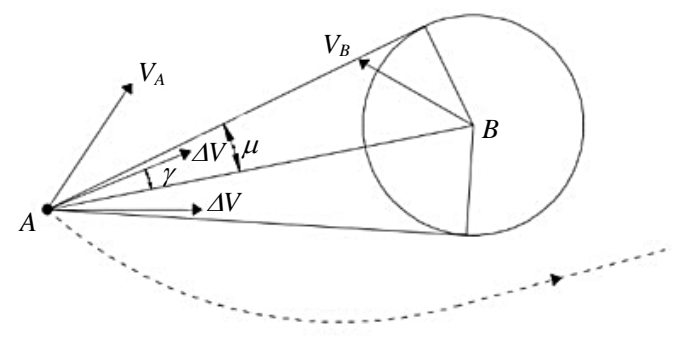

Fig. 11. The strategy of Crossing $(\alpha \geq \phi)$

$$
\begin{cases}\Delta \gamma=-(\mu+\gamma) & \alpha \geq \phi, \phi \geq \theta \\ \Delta \gamma=-(\mu-\gamma) & \alpha \geq \phi, \phi<\theta\end{cases}
$$

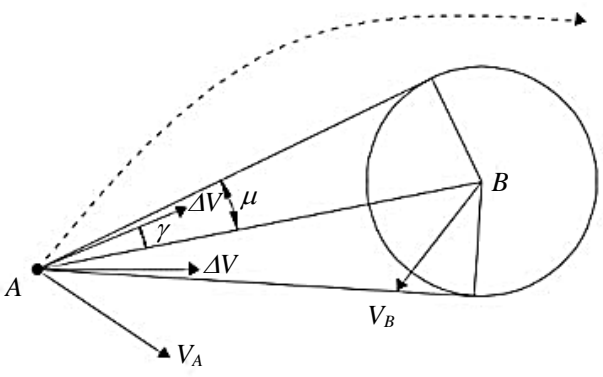

Fig. 12. The strategy of Crossing $(\alpha<\phi)$

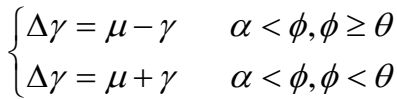

\section{B. Multiple moving obstacles}

When the number of moving obstacles is more than one, it is necessary to calculate the changes of speed and heading angle of collision avoidance relative to each obstacle based on COLREGS. When the heading angle change for different obstacle is inconsistent, it is obviously impossible to make a compound COLREGS collision avoidance action for each obstacle. At this moment, the safety of USV is taken as the first consideration and a collision avoidance strategy with reference obstacle is adopted.

The steps of multiple moving obstacles collision avoidance are as follows:

Step 1: Initialization, assign the USV's velocity and heading angle to $V_{A}$ and $\alpha$, the number of obstacles, $o b s \_n u m$, every obstacle's velocity and heading angle to $V_{B i}$ and $\beta_{i}$.

Step 2: Update the current beat $t, V_{A}$ and $\alpha$, safe_flage $=0$.

Step 3: Calculate the relative velocity, $\Delta V_{i}$, for $O b s_{i}$.

Step 4:Determine whether the collision to $O b s_{i}$ is happened or not. If collision is not happen, go to step 6 .

Step 5:Calculate the changes of velocity, $\Delta V_{A i}$, and heading angle, $\Delta \alpha_{i}$, for collision avoidance of $O b s_{i}$ according to the COLREGS, and safe_flage $=1$.
Step 6: Calculate the next obstacle, $i=i+1$; if $i<$ obs_num, go to step 3.

Step 7: Determine whether the $V_{A}$ and $\alpha$ is safe, if safe_flage $=0$, go to step 10 .

Step 8: Choose the obstacle with the largest change of heading angle $\left(O b s_{\max }\right)$ as the reference obstacle.

Step 9: Calculate new $V_{A}$ and $\alpha$ as Eq. (24).Go to Step 2.

$$
\left\{\begin{array}{l}
V_{A}=V_{A}+\Delta V_{A \max } \\
\alpha=\alpha+\Delta \alpha_{\max }
\end{array}\right.
$$

Step 10: Enter the next beat collision avoidance cycle, output the $V_{A}$ and $\alpha, t=t+1$, and go to step 2 .

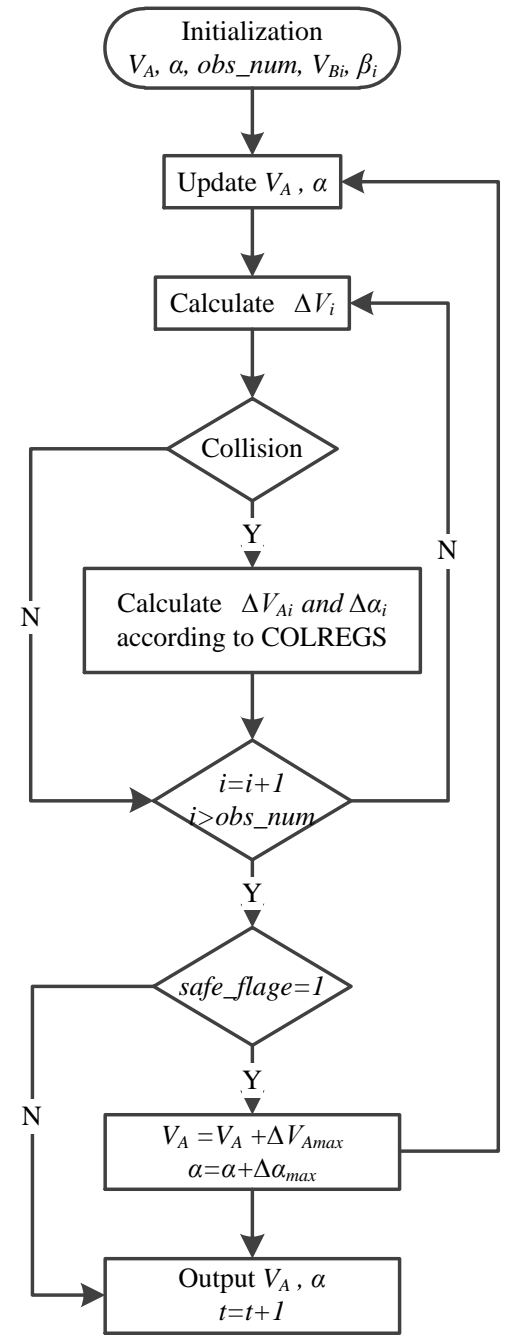

Fig. 13. The steps of collision avoidance for multiple moving obstacles

\section{Simulation Results}

To illustrate the proposed methodology is effective, numerical simulations of USV collision avoidance based on COLREGS are provided. The USV is simplified to a point and the red arrow direction is the current heading angle. The obstacles are ellipses, boundary represents the safety radius. The ' $G$ ' marks the goal point and the ' $S$ ' marks the start point. The blue line is the USV's path and the pink line is the obstacles path. 


\section{A. Simulitiont 1: Single moving obstacle}

The Fig. 14 to Fig. 16 show the simulation results of single moving obstacle avoidance in three encounters are given respectively.

In order to demonstrate the collision avoidance algorithm, an Overtaking scenario is demonstrated first. Fig. 14 (a) shows the original heading angle of USV and obstacle. The velocity and heading angle of USV updated as it receives new information about the obstacle and determines a collision. The algorithm automatically changes the velocity and heading angle to avoid the obstacle in accordance with the COLREGS rules. The local path is constantly updated until the threat of collision has passed, and the USV then headed for the end Goal point. In this case, because of the consideration of the terminal position and driving safety, a starboard overtake is desired as the Fig. 14 (b) shows. The final path (Fig. 14 (c)) shows the entire route taken by both obstacles at the target destination.

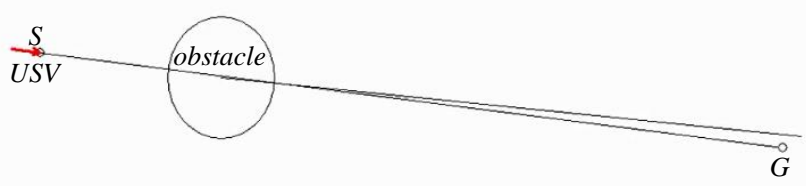

(a) The original heading angle of USV and obstacle

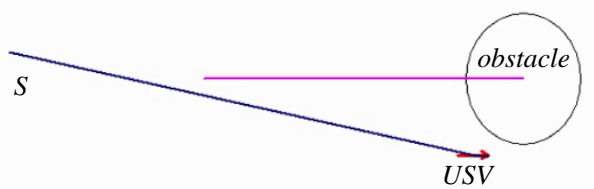

(b) A starboard Overtake in accordance with the COLREGS

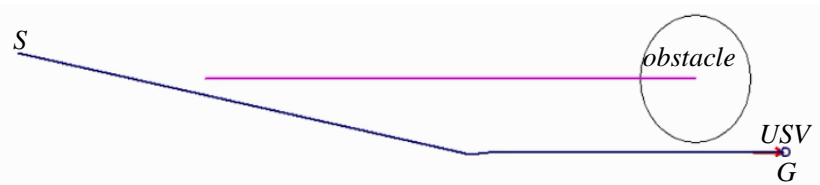

(c) The final path of USV and obstacle.

Fig. 14 The simulation result of a Overtaking collision.

Fig. 15 (a) illustrates a Head-on encounter. Again, when the system detects the oncoming obstacle, a risk assessment is performed. When the potential collision is confirmed, the USV maneuvers starboard to avoid a collision as is shown in Fig. 15(b), while the obstacle maintains its speed and course in accordance with the COLREGS rules. When the obstacle move to the position as shown in Fig. 15(b), no risk of collision is found and the USV then headed for the Goal point. This means the collision has been successfully avoided by taking the proper action. Fig. 15 (c) shows the final path of the USV and obstacle.

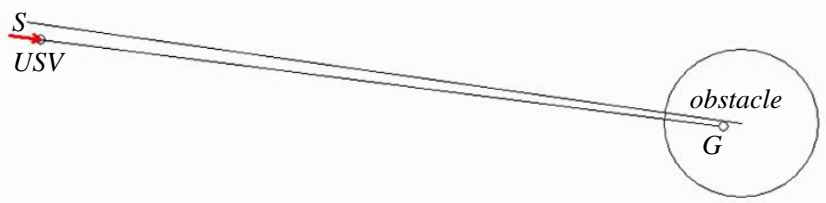

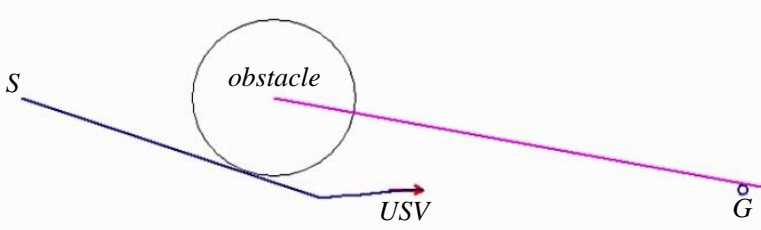

(b) The USV maneuvers starboard to accordance with the COLREGS

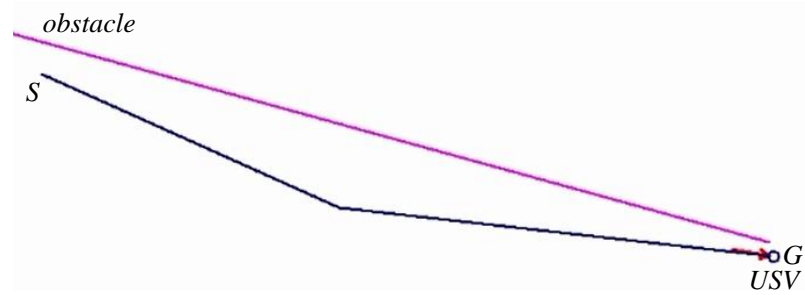

(c) The final path of USV and obstacle.

Fig. 15 The simulation result of a Head-on collision.

Fig. 16 (a) shows the standard Crossing encounter situation, when the system detects that the relative speed of the USV and the obstacle does not exceed the range of the collision angle, the USV maneuvers starboard to avoid a collision as is shown in Fig. 16 (b) while the obstacle maintains its velocity and heading angle in accordance with the COLREGS rules. When the USV move to the position as shown in Fig. 16 (b), USV changes heading angle to the Goal point ,it means the collision has been successfully avoided by taking the proper action. Fig. 16 (c) shows that the USV and obstacle pass each other safely and the final driving path of USV and obstacle.

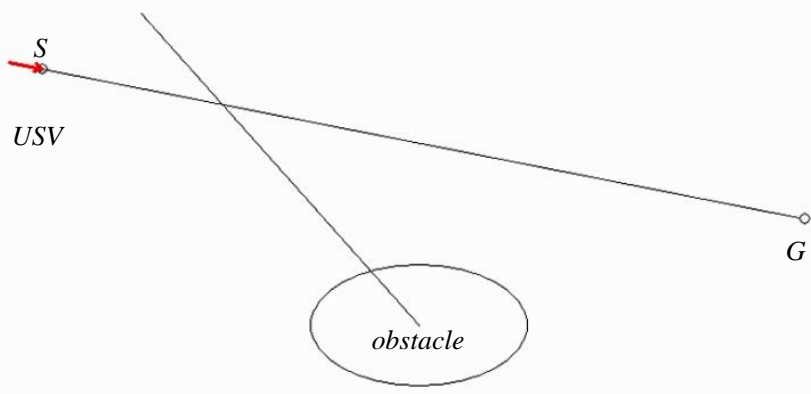

(a) The original heading angle of USV and obstacle.

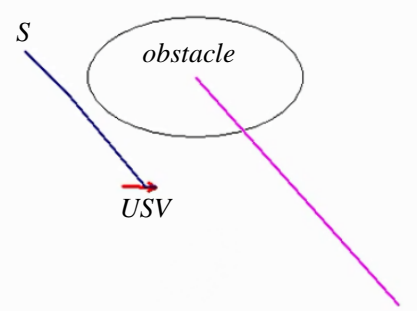

(b) The USV maneuvers starboard to accordance with the COLREGS 


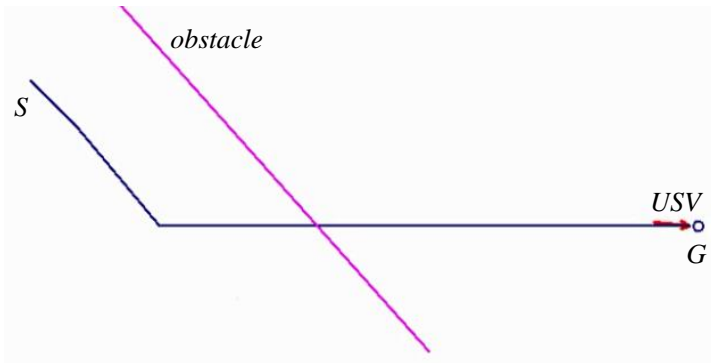

(c) The final path of USV and obstacle.

Fig. 16 The simulation result of a Crossing collision

\section{B. Simulitiont 2: Multiple moving obstacles}

As shown in Fig. 17, a complex traffic scenario is simulated to validate the performance of the proposed approach. The initial conditions of the obstacles involved are illustrated in Fig. 17 (a), the standard encounter situation information listed in the COLREGS. For instance, the USV is involved in a crossing collision with obstacle 1 and obstacle 2 , as well as in a head-on encounter with obstacle 3 . Fig. (b) shows the first time that the USV detects a potential collision and obstacle 1 has the highest priority in terms of collision avoidance. In this configuration, obstacle 1 crosses the USV from the left, and once the collision risk is confirmed, the USV immediately maneuvers port side in accordance with the COLREGS so as to avoid cutting in front of obstacle 1. Fig. (c) illustrates that USV safely passed each of the obstacles involved and moved toward the Goal point. Fig. 17 (d) shows the driving path of USV and the three obstacles.

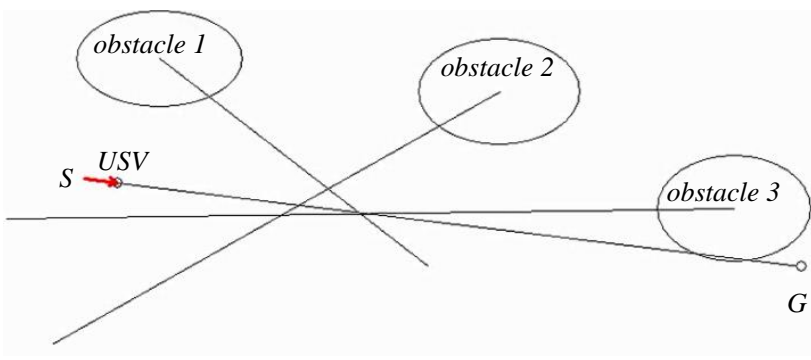

(a) The original heading angle of USV and obstacles.

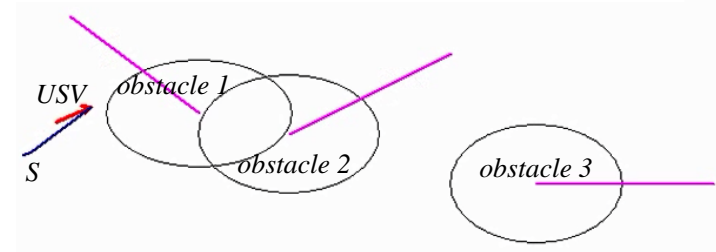

(b) The USV maneuvers port to accordance with the COLREGS

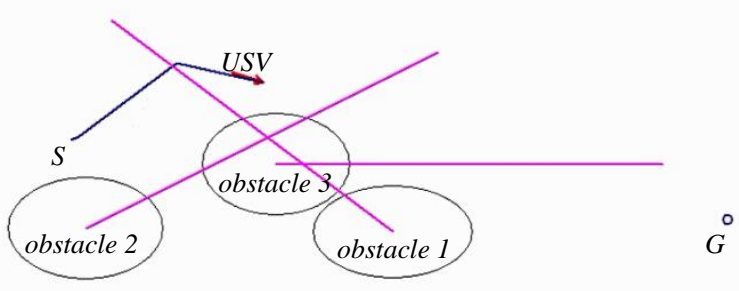

(c) The USV moved to the Goal point

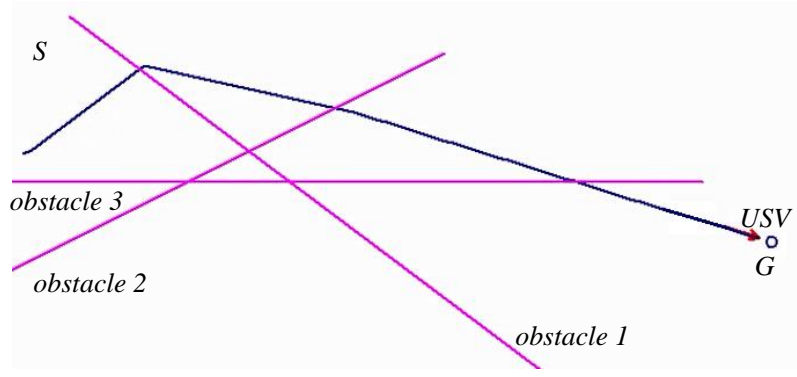

(d) The final path of USV and obstacles.

Fig. 17 The simulation result of a multiple moving obstacles collision

Fig. 17 shows that the proposed approach can interpret the encounter situation correctly and make a decision that is appropriate as well as prompt to avoid collision at the same time as maintaining compliance with the COLREGS rules.

\section{CONCLUSIONS}

An approach for solving the real-time collision avoidance by being compliant with the COLREGS rules for USVs was presented in this paper in order to take full advantage of USV in both military and civic applications. Collision situation is assessed first of all to change the velocity and heading angle of USV in order to complete the collision avoidance of the obstacle. A number of simulations were carried out to confirm the validity of the proposed approach. The results of the simulation indicate that the proposed approach is both valid and efficient; constituting a method that would effectively promote the wide application of USV. In future work, the environment conditions and the maneuverability of USV will be taken into consideration when making a collision avoidance decision, for instance, the wind, wave and the turning radius. As under some circumstances, they can be taken full advantage to reduce the energy consumption to a large extent.

\section{ACKNOWLEDGMENT}

We would like to thank the National Natural Science Foundation of China (Grant No. 51409054), the Foundation Research Funds for the Central Universities (Grant No. HEUCF180101) and High-tech Ships Technology of Ministry of Industry and Information (Grant No. [2016]26) for their financial support of our research.

\section{REFERENCES}

[1] W. Naeem, G.W. Irwin, A. Yang, " COLREGs-based collision avoidance strategies for unmanned surface vehicles", Mechatronics, vol. 22 (6) , pp. 669-678, 2012.

[2] S. Campbell, W. Naeem, G.W. Irwin, "A review on improving the autonomy of unmanned surface vehicles through intelligent collision avoidance maneuver", Annual Reviews in Control, vol. 36 (23) , pp. 267-283, 2012

[3] Navy US. The Navy Unmanned Surface Vehicle (USV) Master Plan, 2007. URL: 〈http://www.navy.mil/navydata/technology/usvmppr〉.

[4] P. Tomas, "Neural anti-collision system for autonomous surface vehicle," Neurocomputing, vol. 149, pp. 559-572, 2015.

[5] Liu, Y. , Liu, W. , Song, R. , \& Bucknall, R. . "Predictive navigation of unmanned surface vehicles in a dynamic maritime environment when using the fast marching method". International Journal of Adaptive Control and Signal Processing, vol. 31(4), pp.464-488, 2015.

[6] P. Svec, A.Thakur, B.C. Shah, S.K. Gupta, "USV trajectory planning for time varying motion goals in an environment with obstacles", ASME 2012 International Design Engineering Technical Conferences and Computers and Information in Engineering Conference, American Society of Mechanical Engineers, Chicago, illinois, USA, 2012, pp. 1297-1306 
[7] R.A. Soltan, H. Ashrafiuon, K.R. Muske, "ODE-based obstacle avoidance and trajectory planning for unmanned surface vessels", Robotica , vol. 29 (5) , pp. 691-703, 2011.

[8] T. Phanthong, T. Maki, T. Ura, T. "Sakamaki, Application of An algorithm for realtime path re-planning of an unmanned surface vehicle avoiding underwater obstacles", Journal of Marine Science and Application, vol. 13 (1) , pp. 105-116, 2014.

[9] U.G.C. Commandant. International Regulations for Preventing Collisions at Sea, (COLREGs), http://www.imo.org/About/Conventions/ListOfConventions/ Pages/COLREG.aspx, 1972.

[10] Campbell S, Naeem W and Irwin G. "A review on improving the autonomy of unmanned surface vehicles through intelligent collision avoidance manoeuvres". Annual Reviews in Control, vol. 36(2), pp. 267-283,2012.

[11] T. Statheros, G. Howells, K.M.D. Maier, "Autonomous ship collision avoidance navigation concepts, technologies and techniques", The Journal of Navigation,, vol. 61 (1), pp. 129-142, 2008.

[12] M.R. Benjamin, J.J. Leonard, J.A. Curcio, P.M. Newman, “A method for protocol - based collision avoidance between autonomous marine surface craft", Journal of Field Robotics, vol. 23 (5) , pp. 333-346, 2006.

[13] C.K. Tam, R. Bucknall, "Cooperative path planning algorithm for marine surface vessels”, Ocean Engineering, vol. 57, pp. 25-33, 2013.

[14] S. Campbell, M. Abu-Tair, W. Naeem, "An automatic COLREGscompliant obstacle avoidance system for an unmanned surface vehicle", Proceedings of the Institution of Mechanical Engineers, Part M: Journal of Engineering for the Maritime Environment Vol 228(2), 2013.

[15] M. Breitsprecher, "Induction of decision rules for the collision regulations", Journal of Theoretical \& Applied Computer Science, vol. 7 (1) , pp. 36-45, 2013.

[16] L.P. Perera, J.P. Carvalho, C. Guedes Soares, "Intelligent ocean navigation and fuzzy Bayesian decision/action formulation", IEEE Journal of Oceanic Engineering, vol 37 (2) , pp. 204-219, 2012.

[17] Zhao Y, Li W, Shi P . "A real-time collision avoidance learning system for Unmanned Surface Vessels". Neurocomputing. Vol.182, pp.255-266, 2016.

[18] Y. Kuwata, M.T. Wolf, D. Zarzhitsky, T.L. Huntsberger, "Safe maritime autonomous navigation with COLREGS, using velocity obstacles", IEEE Journal of Oceanic Engineering, vol. 39 (1) , pp. 110119, 2014.

[19] P. Fiorini, Z. Shiller, "Motion planning in dynamic environments using velocity obstacles", International Journal of Robotics Research, vol. 17 (7) , pp.760-772, 1998.

[20] Tam C K , Bucknall R . "Cooperative path planning algorithm for marine surface vessels". Ocean Engineering, vol. 57 , pp.25-33, 2013.

[21] J Zhuang, L Zhang, S Zhao et al. "Radar-based collision avoidance for unmanned surface vehicles". China Ocean Engineering, vol. 30(6), pp. 867-883, 2016. 